\title{
Fault-tolerant Control of Discrete-time Descriptor Systems using Virtual Actuators
}

\author{
Ye Wang ${ }^{1}$, Damiano Rotondo ${ }^{2}$, Vicenç Puig ${ }^{2}$, Gabriela Cembrano ${ }^{2,3}$ and Yuxin Zhao ${ }^{1}$
}

\begin{abstract}
This paper proposes a fault-tolerant control (FTC) strategy based on virtual actuators for discrete-time descriptor systems subject to multiplicative and additive actuator faults. The goal of this FTC strategy is to reconfigure the closed-loop system by means of a virtual actuator that allows maintaining the use of the nominal controller without having to retune it. To achieve this goal, we define the structure of a dynamical virtual actuator for descriptor systems including multiplicative and additive actuator faults. In order to use a state-feedback controller for implementing the FTC strategy, a state observer for descriptor systems is also defined. Under the proposed FTC scheme, the separation principle of the virtual actuator, the nominal closed-loop system and the state observer is established. Therefore, all the gains can be designed separately. Finally, a numerical example is provided to show the effectiveness of the proposed FTC strategy.
\end{abstract}

\section{INTRODUCTION}

Descriptor systems [1], also known as singular systems [2], [3] or differential-algebraic equation (DAE) systems [4], appear in a wide range of fields, such as water distribution networks [5], aircrafts [6], robotics [7], electrical circuits [8] and biological systems [9], among others. In a descriptor system, some state variables are related algebraically and, consequently, the mathematical formulation is a set of coupled differential and algebraic equations, which include information on both the static and the dynamical constraints of a plant [10]. Descriptor systems have been investigated thoroughly during the last decades, and several results of theoretical and practical relevance have been obtained, such as stabilization [11], pole assignment [12], linear-quadratic optimal control [13] and observer design [14].

Recently, the high demand of reliability, safety and fault tolerance have motivated research in fault diagnosis and fault tolerant control (FTC) [15], [16], [17]. Fault diagnosis of descriptor systems has been performed using zonotopic fault estimation techniques [18], [19], multi-models approaches [20], unknown input observers [21] and $\mathcal{H}_{-} / \mathcal{H}_{\infty}$ observers [22], among others. On the other hand, fewer works about FTC of descriptor systems can be found, which include passive

${ }^{1}$ Y. Wang and Y. Zhao are with College of Automation at Harbin Engineering University, No. 145 Nantong Street, Nangang District, Harbin, P.R. China. E-mail: yewang@hrbeu.edu.cn, zhaoyuxinahrbeu. edu. cn

${ }^{2}$ D. Rotondo, V. Puig and G. Cembrano are with Advanced Control Systems (SAC) Research Group at Institut de Robòtica i Informàtica Industrial (IRI), CSIC-UPC, Universitat Politècnica de Catalunya-Barcelona (UPC), C/. Llorens i Artigas 4-6. 08028 Barcelona, Spain. E-mail: damiano.rotondodyahoo.it, vicenc.puig@upc.edu, cembranodiri.upc.edu

${ }^{3}$ G. Cembrano is also with CETaqua, Water Technology Centre, Ctra d'Esplugues 75, Cornellà de Llobregat, 08940 Barcelona, Spain. approaches, where the controller is pre-designed to be robust against a set of faults [23], and active approaches, where some estimated information about the fault is used online to achieve fault tolerance [24].

In recent years, the fault-hiding paradigm has been proposed as an active FTC strategy to obtain fault tolerance [25]. In this paradigm, the faulty plant is reconfigured by inserting a reconfiguration block, when the fault occurs, named virtual actuator in the case of actuator faults. The virtual actuator aims at hiding the faults from the controller point of view, such that it sees approximately the same plant as before the fault. This active FTC strategy has been extended successfully to many classes of systems, e.g. linear parameter varying (LPV) systems [26], hybrid systems [27], Takagi-Sugeno systems [28], piecewise affine systems [29] and uncertain systems [30]. To the best of our knowledge, this approach has not been extended yet to descriptor systems, which are often used to model cyber-physical systems and other critical infrastructures, such as water distribution networks [5]. To maintain the safety of such systems, an FTC strategy is required.

The main contribution of this paper is to propose a virtualactuator-based FTC strategy for discrete-time linear descriptor systems. The detailed contributions are summarized as follows:

- Partly based on the work [26], we define the novel structure of the virtual actuator for descriptor systems by considering both multiplicative and additive actuator faults. The FTC strategy adds the virtual actuator reconfiguration block to the nominal closed-loop system, which comprises the nominal state-feedback controller and the state observer;

- We present the separation principle for the augmented system made up by the virtual actuator and the nominal closed-loop system, which means that the admissibility of the virtual actuator can be analyzed on its own.

- We transform the admissibility and stability conditions to linear matrix inequalities (LMIs), which can be solved efficiently using available solvers.

The remainder of this paper is structured as follows. The problem statement is formulated in Section II. The main results including the structure of the virtual actuator for descriptor systems and the FTC strategy are presented in Section III. An illustrative example is provided in Section IV. Finally, the conclusion and some future remarks are addressed in Section V.

Notation: We use $I_{r}$ to denote an identity matrix with dimension $r$. Note that the dimension $r$ of $I$ may be dropped 
when it can be inferred. For a matrix $A$, we use $\operatorname{rank}(A)$, $A^{T}$ and $A^{\dagger}$ to denote the rank, the transpose, and the pseudoinverse of $A$, respectively. We denote $\operatorname{He}(A)=A+A^{T}$. $A \succ 0(A \prec 0)$ denotes positive (negative) definiteness. For two matrices $E$ and $A$, we denote the set of generalized eigenvalues as $\sigma(E, A)=(E, A) \in\{\sigma: \operatorname{det}(\sigma E-A)=$ $0\}$, and $\sigma(A)$, obtained with $E=I$, denotes the set of eigenvalues of $A$. For a vector $z$, we use $\operatorname{diag}(z)$ to denote a diagonal matrix with diagonal elements given by $z$.

\section{Problem Statement}

Consider the class of discrete-time linear time-invariant descriptor systems as

$$
\begin{aligned}
E x(k+1) & =A x(k)+B u(k), \\
y(k) & =C x(k),
\end{aligned}
$$

where $x \in \mathbb{R}^{n}, u \in \mathbb{R}^{m}$ and $y \in \mathbb{R}^{p}$ denote the system state, control input and measurement output vectors, respectively. $A \in \mathbb{R}^{n \times n}, B \in \mathbb{R}^{n \times m}$ and $C \in \mathbb{R}^{p \times n}$ are the system matrices. By definition of descriptor systems, the matrix $E \in$ $\mathbb{R}^{n \times n}$ is singular, that is $\operatorname{rank}(E)=r<n$.

Lemma 1 ([2]): For the pair $(E, A)$ of the descriptor system (1),

- Regularity: the pair $(E, A)$ is regular if $\operatorname{det}(z E-A)$ is not identically zero.

- Causality: the pair $(E, A)$ is causal if $\operatorname{deg}(\operatorname{det}(z E-A))=\operatorname{rank}(E)$.

- Stability: the pair $(E, A)$ is stable if $|\sigma(E, A)|<1$.

- Admissibility: the pair $(E, A)$ is admissible if it is regular, causal and stable.

In this paper, the descriptor system (1) is assumed to be observable and matrices $E$ and $C$ satisfy the rank condition $\operatorname{rank}\left[\begin{array}{c}E \\ C\end{array}\right]=n$. Therefore, there exists a pair of $T \in \mathbb{R}^{n \times n}$ and $N \in \mathbb{R}^{n \times p}$ such that

$$
T E+N C=I .
$$

When actuator faults occur, the descriptor system (1) becomes

$$
\begin{aligned}
E x_{f}(k+1) & =A x_{f}(k)+B_{f}(\phi(k))\left(u_{f}(k)+f(k)\right), \\
y_{f}(k) & =C x_{f}(k),
\end{aligned}
$$

where $x_{f} \in \mathbb{R}^{n}, u_{f} \in \mathbb{R}^{m}$ and $y_{f} \in \mathbb{R}^{p}$ denote the faulty system state, control input and measurement output vectors, respectively. $f \in \mathbb{R}^{m}$ denotes the additive actuatorfault vector. Besides, the multiplicative actuator-fault vector $\phi(k) \in \mathbb{R}^{m}$ is embedded in $B_{f}(\phi(k)) \in \mathbb{R}^{n \times m}$ as

$$
B_{f}(\phi(k))=B \operatorname{diag}\left(\phi_{1}(k), \ldots, \phi_{m}(k)\right),
$$

with $0 \leq \phi_{i}(k) \leq 1, i=1, \ldots, m$.

In the following, we will consider that the additive and multiplicative actuator faults are available using a suitable fault estimation technique, e.g. as in [31]. We focus on designing a virtual actuator for the reconfiguration of the faulty system (3) and its application to FTC of the descriptor system (3) using a nominal observer-based state-feedback controller.

\section{MAin Results}

We now propose the FTC strategy based on virtual actuators for the descriptor system (3). The general FTC scheme is shown in Fig 1.

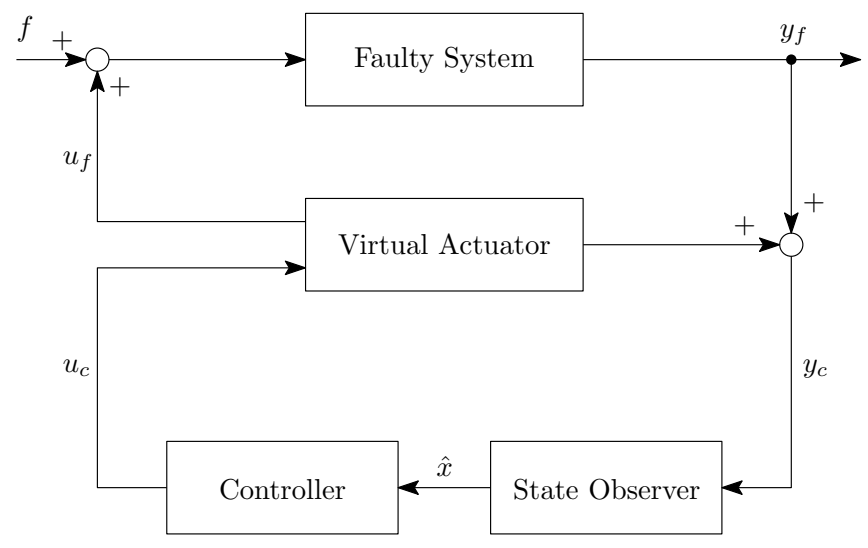

Fig. 1. Virtual-actuator-based FTC Scheme.

Denote the nominal input and output vectors as $u_{c} \in \mathbb{R}^{m}$ and $y_{c} \in \mathbb{R}^{p}$, respectively. We consider an observer-based state-feedback control strategy as

$$
u_{c}(k)=K \hat{x}(k),
$$

where $\hat{x} \in \mathbb{R}^{n}$ is the estimated state and $K \in \mathbb{R}^{m \times n}$ is the state-feedback gain.

\section{A. Descriptor State Observer}

Based on the nominal descriptor system (1), the state observer is structured as follows:

$$
\begin{aligned}
z(k+1) & =(T A-L C) \hat{x}(k)+T B u_{c}(k)+L y_{c}(k), \\
\hat{x}(k) & =z(k)+N y_{c}(k),
\end{aligned}
$$

where $z \in \mathbb{R}^{n}$ and $\hat{x} \in \mathbb{R}^{n}$ denote the observer state and the estimated state, respectively. Besides, $L \in \mathbb{R}^{n \times p}$ is the state observer gain.

\section{B. Virtual Actuator for Descriptor Systems}

Based on [26], let us define

$$
\begin{aligned}
N_{v a}(\phi(k)) & =B_{f}(\phi(k))^{\dagger} B, \\
B^{*} & =B_{f}(\phi(k)) N_{v a}(\phi(k)) .
\end{aligned}
$$

According to [16, Appendix B], it is noticed that $B^{*}$ does not depend on $\phi(k)$.

Therefore, the virtual actuator structure for the fault descriptor system (3) is defined in the following.

Definition 1 (Virtual actuator for descriptor systems):

Given the faulty descriptor system (3) with multiplicative and additive actuator faults, the virtual actuator is structured as follows:

$$
\begin{aligned}
E x_{v a}(k+1) & =\left(A+B^{*} M_{v a}\right) x_{v a}(k)+\left(B-B^{*}\right) u_{c}(k), \\
u_{f}(k) & =N_{v a}(\phi(k))\left(u_{c}(k)-M_{v a} x_{v a}(k)\right)-f(k), \\
y_{c}(k) & =y_{f}(k)+C x_{v a}(k),
\end{aligned}
$$

where $x_{v a} \in \mathbb{R}^{n}$ is the virtual actuator state and $M_{v a} \in$ $\mathbb{R}^{m \times n}$ is the virtual actuator gain. 


\section{Separation principle}

As shown in Fig. 1, the closed-loop virtual-actuatorbased FTC scheme is presented. A virtual actuator is set up between the faulty system and the state-feedback controller (with the observer). In this case, the controller can be designed using the nominal model. The actual control input will be compensated by the designed virtual actuator. In the following, we first discuss the behavior of this closed-loop system.

Theorem 1: Consider the faulty descriptor (3), the statefeedback controller in (4), the state observer in (5), and the virtual actuator in (7), and define the new variables $x_{1}(k)=$ $x_{v a}(k), x_{2}(k)=x_{f}(k)+x_{v a}(k)$ and $x_{3}(k)=\hat{x}(k)-x_{f}(k)-$ $x_{v a}(k)$. Then, the closed-loop behavior is given by

$$
E_{v a}\left[\begin{array}{l}
x_{1}(k+1) \\
x_{2}(k+1) \\
x_{3}(k+1)
\end{array}\right]=A_{v a}\left[\begin{array}{l}
x_{1}(k) \\
x_{2}(k) \\
x_{3}(k)
\end{array}\right]
$$

where

$$
\begin{gathered}
E_{v a}=\left[\begin{array}{ccc}
E & 0 & 0 \\
0 & E & 0 \\
0 & 0 & I_{n}
\end{array}\right], \\
A_{v a}=\left[\begin{array}{ccc}
A+B^{*} M_{v a} & \left(B-B^{*}\right) K & \left(B-B^{*}\right) K \\
0 & A+B K & B K \\
0 & 0 & (T A-L C) \\
\text { Proof: Considering the defined new variables, it follows }
\end{array}\right.
\end{gathered}
$$

Proof: Considering the defined new variables, it follows

$$
\begin{aligned}
x_{v a}(k) & =x_{1}(k), \\
x_{f}(k) & =-x_{1}(k)+x_{2}(k), \\
\hat{x}(k) & =x_{2}(k)+x_{3}(k) .
\end{aligned}
$$

Taking into account the dynamics of the virtual actuator in (7), we have

$$
\begin{aligned}
E x_{1}(k+1)= & \left(A+B^{*} M_{v a}\right) x_{1}(k)+\left(B-B^{*}\right) K x_{2}(k) \\
& +\left(B-B^{*}\right) K x_{3}(k) .
\end{aligned}
$$

Together with the dynamics of (3), we derive

$$
\begin{aligned}
E x_{2}(k+1) & =E x_{f}(k+1)+E x_{v a}(k+1) \\
& =(A+B K) x_{2}(k)+B K x_{3}(k) .
\end{aligned}
$$

With $T$ and $N$ satisfying (2), the dynamics of the descriptor observer (5) can be reformulated as

$$
\begin{aligned}
\hat{x}(k+1)= & (T A-L C) \hat{x}(k)+T B u_{c}(k) \\
& +L y_{c}(k)+N y_{c}(k+1) .
\end{aligned}
$$

Therefore, we derive

$$
\begin{aligned}
x_{3}(k+1)= & \hat{x}(k+1)-x_{f}(k+1)-x_{v a}(k+1) \\
= & z(k+1)+N C x_{f}(k+1)+N C x_{v a}(k+1) \\
& -x_{f}(k+1)-x_{v a}(k+1) \\
= & z(k+1)-T E x_{f}(k+1)-T E x_{v a}(k+1) \\
= & (T A-L C) \hat{x}(k)+T B u_{c}(k) \\
& +L\left(y_{f}(k)+C x_{v a}(k)\right) \\
& -T\left(E x_{f}(k+1)+E x_{v a}(k+1)\right) \\
= & (T A-L C) x_{3}(k) .
\end{aligned}
$$

By reformulating the closed-loop behaviour of controller, observer and virtual actuator in a augmented form, we obtain (8).

From the closed-loop behavior in Theorem 1, we now present the separation principle for the design of the virtual actuator, the state-feedback controller and the state observer of the descriptor system (3).

Theorem 2 (Separation principle): Consider the closedloop descriptor system (8), the pair $\left(E_{v a}, A_{v a}\right)$ is admissible if and only if the pairs $\left(E, A+B^{*} M_{v a}\right)$ and $(E, A+B K)$ are admissible and the matrix $(T A-L C)$ is Schur stable.

Proof: From (8), $A_{v a}$ is a upper-triangle block matrix. Regularity is satisfied since $\operatorname{det}\left(z E_{v a}-A_{v a}\right)=\operatorname{det}(z E-$ $\left.\left.\left(A+B^{*} M_{v a}\right)\right) \operatorname{det}(z E-(A+B K)) \operatorname{det}(z I-(T A-L C))\right)$ is not identically zero due to the hypothesis of admissibility of the pairs $\left(E, A+B^{*} M v a\right)$ and $(E, A+B K)$. Regarding causality, since $z E_{v a}-A_{v a}$ also leads to a upper-triangle block matrix and it satisfies $\operatorname{deg}\left(\operatorname{det}\left(z E_{v a}-A_{v a}\right)\right)=$ $\operatorname{rank}\left(E_{v a}\right)=2 \operatorname{rank}(E)+n$. Furthermore, the set of generalized eigenvalues $\sigma\left(E_{v a}, A_{v a}\right)$ of the reconfigured closed-loop system (8) consists of the set of generalized eigenvalues $\sigma\left(E, A+B^{*} M_{v a}\right)$ of the virtual actuator (7), the set of generalized eigenvalues $\sigma(E, A+B K)$ of the nominal closed-loop system (1)-(4), and the set of eigenvalues $\sigma(T A-L C)$ of the state observer, that is,

$$
\begin{aligned}
\sigma\left(E_{v a}, A_{v a}\right)= & \sigma\left(E, A+B^{*} M_{v a}\right) \cup \sigma(E, A+B K) \\
& \cup \sigma(T A-L C) .
\end{aligned}
$$

Thus, the admissibility of $\left(E_{v a}, A_{v a}\right)$ is stated based on Lemma 1.

\section{Closed-loop Design}

Based on the separation principle proposed in Theorem 2, the gains $M_{v a}, K$ and $L$ can be designed separately such that the admissibility of the reconfigured closed-loop system can be guaranteed.

Lemma 2 ([3]): Given an autonomous descriptor system $E x(k+1)=A x(k)$, the pair $(E, A)$ is admissible if and only if there exist matrices $P \in \mathbb{R}^{n \times n} \succ 0$ and $Q \in$ $\mathbb{R}^{n \times(n-r)}$ such that

$$
A^{T} P A-E^{T} P E+\mathbf{H e}\left(Q S^{T} A\right) \prec 0,
$$

where $S \in \mathbb{R}^{n \times(n-r)}$ is any matrix with full-column rank satisfying $E^{T} S=0$.

Proof: The proof of this sufficient and necessary admissibility condition of discrete-time descriptor systems can be found in [3, Theorem 2.4].

Based on Lemma 2, we now present the admissibility conditions for the virtual actuator (7) and the nominal controller (4)

Theorem 3: Given the faulty descriptor system (3), the pair $\left(E, A+B^{*} M_{v a}\right)$ of the virtual actuator (7) is admissible if there exist matrices $P_{1}=P_{1}^{T} \in \mathbb{R}^{n \times n}$ with $P_{1} \succ 0, Q_{1} \in$ $\mathbb{R}^{n \times(n-r)}$ and $M_{v a} \in \mathbb{R}^{m \times n}$ such that

$$
\left[\begin{array}{cc}
\Psi_{1} & \left(A+B^{*} M_{v a}\right)^{T} P_{1} \\
P_{1}\left(A+B^{*} M_{v a}\right) & -P_{1}
\end{array}\right] \prec 0,
$$


where $\Psi_{1}=-E^{T} P_{1} E+\mathbf{H e}\left(Q_{1} S^{T}\left(A+B^{*} M_{v a}\right)\right)$ and $S \in$ $\mathbb{R}^{n \times(n-r)}$ with full-column rank satisfies $E^{T} S=0$.

Proof: Consider the system $\operatorname{Ex}(k+1)=(A+$ $\left.B^{*} M_{v a}\right) x(k)$. Based on Lemma 2, the pair $\left(E, A+B^{*} M_{v a}\right)$ is admissible if there exist matrices $P_{1} \in \mathbb{R}^{n \times n} \succ 0, Q_{1} \in$ $\mathbb{R}^{n \times(n-r)}$ and $M_{v a} \in \mathbb{R}^{m \times n}$ such that

$$
\begin{aligned}
(A & \left.+B^{*} M_{v a}\right)^{T} P_{1}\left(A+B^{*} M_{v a}\right)-E^{T} P_{1} E \\
& +\operatorname{He}\left(Q_{1} S^{T}\left(A+B^{*} M_{v a}\right)\right) \prec 0 .
\end{aligned}
$$

By applying the Schur complement to the above inequality, we thus obtain (10).

Theorem 4: Given the nominal descriptor system (1), the pair $(E, A+B K)$ of the nominal controller is admissible if there exist matrices $P_{2} \in \mathbb{R}^{n \times n} \succ 0, Q_{2} \in \mathbb{R}^{n \times(n-r)}$ and $K \in \mathbb{R}^{m \times n}$ such that

$$
\left[\begin{array}{cc}
\Psi_{2} & (A+B K)^{T} P_{2} \\
P_{2}(A+B K) & -P_{2}
\end{array}\right] \prec 0,
$$

where $\Psi_{2}=-E^{T} P_{2} E+\mathbf{H e}\left(Q_{2} S^{T}(A+B K)\right)$ and $S \in$ $\mathbb{R}^{(n-r) \times n}$ with full-column rank satisfies $E^{T} S=0$.

Proof: Similar to the proof of Theorem 3, by using the Schur complement, we use the result in (9) for the pair $(E, A+B K)$ to obtain the admissibility condition in (11).

Since the dynamics of the state observer is given in a standard dynamical form, we propose the LMI condition of the asymptotic stability of the state observer as follows.

Theorem 5: Given the nominal descriptor system (1), matrices $T$ and $N$ satisfying (2), the state observer in (5) is asymptotically stable if there exist matrices $W=W^{T} \in$ $\mathbb{R}^{n \times n}, G \in \mathbb{R}^{n \times n}$ and $Y \in \mathbb{R}^{n \times p}$ such that

$$
\left[\begin{array}{cc}
-W & A^{T} T^{T} G^{T}-C^{T} Y^{T} \\
G T A-Y C & W-G-G^{T}
\end{array}\right] \prec 0 .
$$

Proof: Consider the system $x(k+1)=\tilde{A} x(k)$ with $\tilde{A}=T A-L C$. Based on the Lyapunov stability theory, this system is asymptotically stable if there exists a matrix $W=W^{T} \in \mathbb{R}^{n \times n}$ such that $W \succ 0$ and

$$
\tilde{A}^{T} W \tilde{A}-W \prec 0 .
$$

Refer to [32, Theorem 1], the above stability conditions are equivalent to (12). That is, by pre-multiplying $\left[I_{n},-A^{T}\right]$ and post-multiplying its transpose to (12), we thus obtain (13) by introducing $Y=G L$.

By satisfying (12), the gain of the state observer (5) can be computed by $L=G^{-1} Y$.

\section{NUMERICAL EXAMPLES}

\section{A. Example 1}

In order to illustrate the proposed virtual-actuator-based FTC strategy, we first use an example introduced in [3, Example 3.2]. Consider the nominal descriptor system (1) with

$$
\begin{aligned}
& E=\left[\begin{array}{ccc}
1 & 0.8 & 0 \\
0 & 1 & 0 \\
0 & 0.4 & 0
\end{array}\right], A=\left[\begin{array}{ccc}
1.2 & 0.64 & 0 \\
0 & 0.8 & 0.3 \\
0 & 0.32 & 1
\end{array}\right], \\
& B=\left[\begin{array}{ccc}
1 & 1.3 & 2 \\
0 & 1 & 0.24 \\
0 & 0.4 & 0.8
\end{array}\right], C=\left[\begin{array}{ccc}
1 & 0 & 0 \\
0 & 1 & 1
\end{array}\right],
\end{aligned}
$$

and the faulty descriptor system (3) with $f=0$ and $B_{f}=$ $B \operatorname{diag}\left(\left[\phi_{1}, \phi_{2}, \phi_{3}\right]\right)$, where three faulty scenarios are taken into account as follows:

1) Actuator 1 totally lost: $\phi_{1}=0, \phi_{2}=0.6$ and $\phi_{3}=0.3$.

2) Actuator 2 totally lost: $\phi_{1}=0.8, \phi_{2}=0$ and $\phi_{3}=0.3$.

3) Actuator 3 totally lost: $\phi_{1}=0.8, \phi_{2}=0.6$ and $\phi_{3}=0$.

The design has been carried out in MATLAB with YALMIP toolbox [33]. We use the PEMBMI solver [34] to design the virtual actuator and controller by solving the matrix inequalities in (10) and (11), respectively, and the MOSEK solver [35] to design the observer by solving (12). Matrices $T$ and $N$ are selected satisfying condition (2) as follows:

$T=\left[\begin{array}{ccc}0.0135 & -0.2162 & 0.5135 \\ -0.1081 & 0.7297 & 0.8919 \\ -0.6486 & -0.6216 & 0.3514\end{array}\right], N=\left[\begin{array}{cc}0.9865 & 0 \\ 0.1081 & 0 \\ 0.6486 & 1\end{array}\right]$

The obtained virtual actuator designs for the different scenarios are

(i) Scenario 1:

$$
\begin{gathered}
M_{v a}=\left[\begin{array}{ccc}
-0.1049 & -0.2530 & 0.9299 \\
0.1363 & -0.9687 & -0.5400 \\
-0.5861 & 0.4142 & -0.1095
\end{array}\right], \\
\left|\sigma\left(E, A+B^{*} M_{v a}\right)\right|=\{0.1112,0.0087\},
\end{gathered}
$$

(ii) Scenario 2:

$$
\begin{aligned}
& M_{v a}=\left[\begin{array}{ccc}
-1.6359 & 0.7190 & 1.0057 \\
-1.9488 & 2.1347 & 0.3841 \\
1.4731 & -2.0120 & -0.7290
\end{array}\right], \\
& \left|\sigma\left(E, A+B^{*} M_{v a}\right)\right|=\{0.0180,0.8\}
\end{aligned}
$$

(iii) Scenario 3:

$$
\begin{aligned}
& M_{v a}=\left[\begin{array}{ccc}
-0.4354 & 0.2930 & 0.3993 \\
0.2533 & -0.8465 & -0.7258 \\
-0.5994 & 0.0603 & 0.2084
\end{array}\right], \\
& \left|\sigma\left(E, A+B^{*} M_{v a}\right)\right|=\{0.0920,0.0015\} .
\end{aligned}
$$

The designs of $K$ and $L$ are not affected by the actuator faults. Thus, by solving (11) and (12) for the nominal system, the obtained results are

$$
\begin{aligned}
K & =\left[\begin{array}{ccc}
-1.1640 & 0.3546 & 1.0359 \\
0.0117 & -0.8153 & -0.5260 \\
-0.0255 & 0.0326 & -0.1815
\end{array}\right], \\
L & =\left[\begin{array}{cc}
0.0388 & 0.2565 \\
-0.1140 & 0.9777 \\
-0.7297 & -0.2484
\end{array}\right]
\end{aligned}
$$

with $|\sigma(E, A+B K)|=\left\{3.7888 \times 10^{-5}, 0.0012\right\}$, $|\sigma(T A-L C)|=\left\{0.2130,0,3.818 \times 10^{-5}\right\}$. 
From the obtained results above, we can see that the eigenvalues/generalized eigenvalues of the observer, the virtual actuator and the nominal controller are all inside a unit circle in all three scenarios.

\section{B. Example 2}

With the proposed FTC strategy, we would like to show the closed-loop behaviors in simulations. Thus, consider the following descriptor system (1) with

$$
\begin{aligned}
& E=\left[\begin{array}{ccc}
1 & 0.8 & 0 \\
0 & 1 & 0 \\
0 & 0.4 & 0
\end{array}\right], A=\left[\begin{array}{ccc}
2 & 1.2 & 0 \\
0 & 1.5 & 0.3 \\
0 & 0.6 & 1
\end{array}\right], \\
& B=\left[\begin{array}{ccc}
1 & 1.3 & 2 \\
0 & 1 & 0 \\
0 & 0.4 & 0
\end{array}\right], C=\left[\begin{array}{lll}
1 & 0 & 0 \\
0 & 1 & 1
\end{array}\right],
\end{aligned}
$$

and the actuator-fault matrix is given by $B_{f}=$ $B\left[\begin{array}{ccc}0 & 0 & 0 \\ 0 & 0.6 & 0 \\ 0 & 0 & 0.3\end{array}\right]$. The initial state is set to be $x(0)=$ $[5,3,3.5]^{T}$. We have carried out the closed-loop simulation in three scenarios: (i) no actuator-fault; (ii) actuator-fault without using the virtual actuator; (iii) actuator-fault using the virtual actuator. The actuator fault (with $B_{f}$ ) is introduced into the system at the sample $k=5$. With a delay period, we enable the virtual actuator at the sample $k=8$. We also choose $T$ and $N$ by satisfying the condition in (2) to obtain

$$
T=\left[\begin{array}{ccc}
0.5 & 0 & 1 \\
0 & 1 & 1 \\
0 & -1 & 1
\end{array}\right], N=\left[\begin{array}{cc}
0.5 & 0 \\
0 & 0 \\
0 & 1
\end{array}\right]
$$

With the same tools used in Example 1, we obtain the gains for the observer, the virtual actuator and the nominal controller for this system as follows:

$$
\begin{aligned}
M_{v a}= & {\left[\begin{array}{ccc}
-0.8631 & -4.5332 & 0.1009 \\
0.0734 & -1.1160 & -0.0104 \\
-0.6735 & 2.1113 & -0.0351
\end{array}\right], } \\
K= & {\left[\begin{array}{ccc}
-1.3348 & 0.7187 & -3.9558 \\
0.0009 & -1.6001 & -0.0072 \\
-0.3841 & 0.0408 & 1.9893
\end{array}\right], } \\
L= & {\left[\begin{array}{cc}
1.6278 & 1.0278 \\
-0.3167 & 0.9833 \\
1.3722 & 1.1722
\end{array}\right] . }
\end{aligned}
$$

Now, we simulate the closed-loop system for 20 samples. The closed-loop simulation results are shown in Fig. 2-4. From the sample $k=1$ to $k=5$, since there is no fault occurred inside the system, the state trajectories of three cases are the same. From the sample $k=5$ to $k=8$, the actuator faults are inserted in the closed-loop systems. Since the virtual-actuator-based FTC strategy is not enabled, the closed-loop state trajectories in the cases without and with virtual actuator are driving away from the equilibrium points and the closed-loop system becomes unstable. Starting from the sample $k=8$, the virtual actuator based FTC strategy is enabled. Therefore, the closed-loop state trajectories return to the equilibrium point again. As a result, the closed-loop

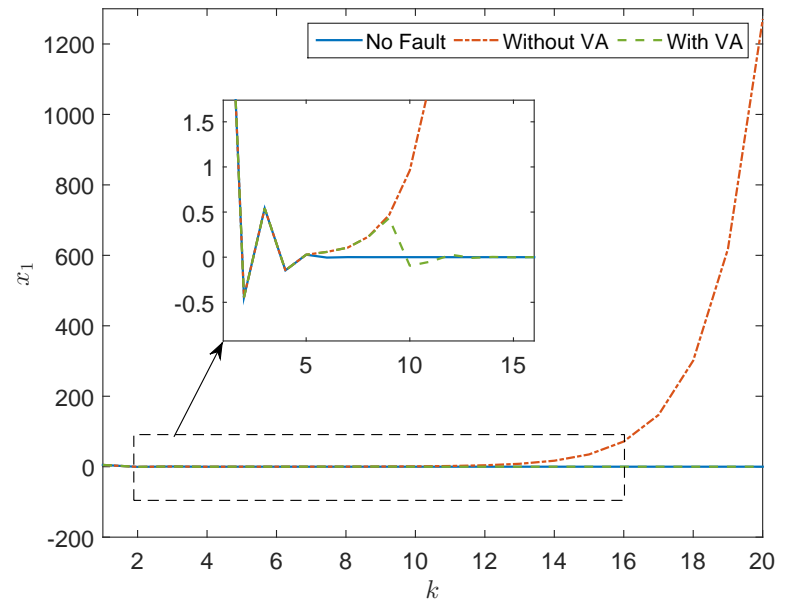

Fig. 2. The simulation result for $x_{1}$.

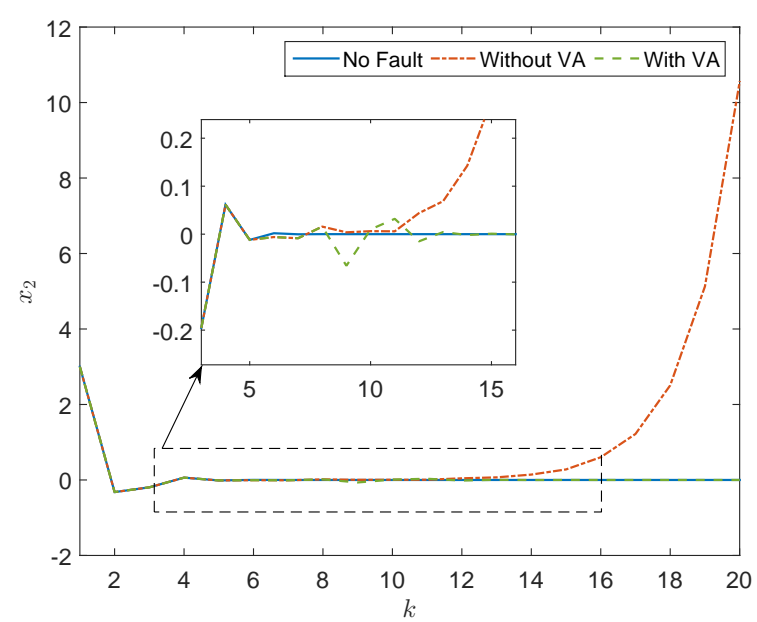

Fig. 3. The simulation result for $x_{2}$.

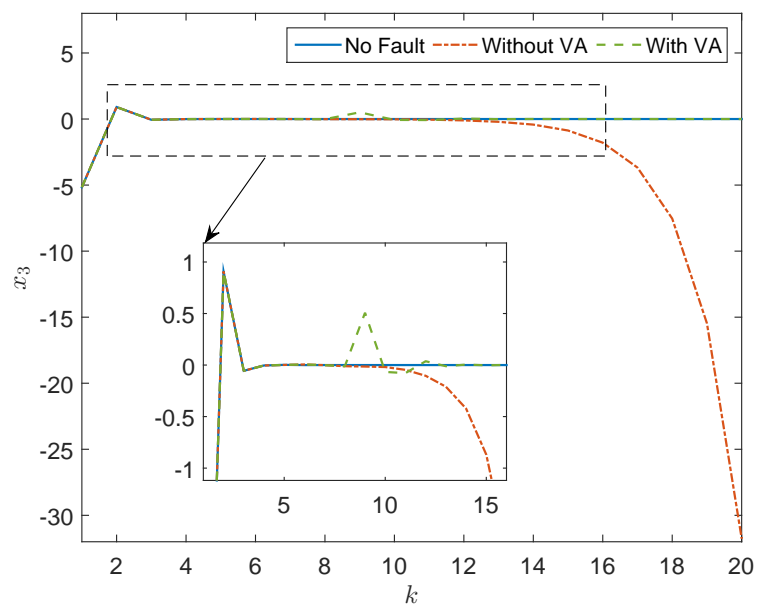

Fig. 4. The simulation result for $x_{3}$. 
system is stable and converges again towards the origin of the state space.

\section{CONCLUSION}

In this paper, we have proposed a virtual-actuator-based FTC strategy for discrete-time descriptor systems. The proposed FTC strategy includes an observer-based nominal state-feedback and a virtual actuator. The separation principle of the closed-loop FTC system has been established. The design of the virtual actuator, the nominal state-feedback controller and the state observer can be done in a independent manner. The closed-loop admissibility is guaranteed by using the admissibility conditions to design the virtual actuator and the nominal state-feedback controller, and the asymptotic stability condition to design the state observer. Some numerical examples have been used to illustrate the proposed approach. As a future work, the proposed FTC strategy will be extended by adding a virtual sensor to handle sensor faults in descriptor systems.

\section{ACKNOWLEDGEMENT}

This work has been partially funded by the Spanish State Research Agency (AEI) and the European Regional Development Fund (ERFD) through the project DEOCS (ref. DPI2016-76493-C3-3-R), the María de Maeztu Seal of Excellence to IRI (MDM-2016-0656), and the grant Juan de la Cierva-Formación (FJCI-2016-29019), and by the Fundamental Research Funds for the Central University (No. 3072019CFJ0406).

\section{REFERENCES}

[1] G.-R. Duan, Analysis and design of descriptor linear systems. Springer Science \& Business Media, 2010, vol. 23.

[2] L. Dai, Singular Control Systems. Berlin Heidelberg, Germany: Springer, 1989.

[3] S. Xu and J. Lam, Robust Control and Filtering of Singular Systems. Springer, 2006.

[4] A. Kumar and P. Daoutidis, Control of nonlinear differential algebraic equation systems with applications to chemical processes. CRC Press, 1999, vol. 397.

[5] Y. Wang, V. Puig, and G. Cembrano, "Non-linear economic model predictive control of water distribution networks," Journal of Process Control, vol. 56, pp. 23-34, 2017.

[6] I. Masubuchi, J. Kato, M. Saeki, and A. Ohara, "Gain-scheduled controller design based on descriptor representation of LPV systems: Application to flight vehicle control," in Decision and Control, 2004. CDC. 43rd IEEE Conference on, vol. 1. IEEE, 2004, pp. 815-820.

[7] L. Vermeiren, A. Dequidt, M. Afroun, and T.-M. Guerra, "Motion control of planar parallel robot using the fuzzy descriptor system approach," ISA transactions, vol. 51, no. 5, pp. 596-608, 2012.

[8] L. Sun, Y. Wang, and G. Feng, "Control design for a class of affine nonlinear descriptor systems with actuator saturation," IEEE transactions on automatic control, vol. 60, no. 8, pp. 2195-2200, 2015.

[9] P. Liu, Q. Zhang, X. Yang, and L. Yang, "Passivity and optimal control of descriptor biological complex systems," IEEE Transactions on Automatic Control, vol. 53, no. Special Issue, pp. 122-125, 2008.

[10] S. Xu and J. Lam, Robust control and filtering of singular systems, 2006.

[11] T. Kaczorek, "Stabilization of linear descriptor systems by statefeedback controllers," Applied Mathematics and Computer Science, vol. 6, pp. 27-32, 1996.

[12] S.-Y. Zhang, "Pole placement for singular systems," Systems \& control letters, vol. 12, no. 4, pp. 339-342, 1989.
[13] D. Rotondo, F. Nejjari, and V. Puig, "Shifting linear quadratic control of constrained continuous-time descriptor LPV systems," in 1st IFAC Workshop on Linear Parameter Varying Systems, Grenoble, France, 2015.

[14] M. Hou and P. Muller, "Observer design for descriptor systems," IEEE Transactions on Automatic Control, vol. 44, no. 1, pp. 164-169, 1999.

[15] Y. Zhang and J. Jiang, "Bibliographical review on reconfigurable faulttolerant control systems," Annual reviews in control, vol. 32, no. 2, pp. 229-252, 2008.

[16] D. Rotondo, Advances in Gain-Scheduling and Fault Tolerant Control Techniques. Springer, 2018.

[17] M. Witczak, "Fault diagnosis and fault-tolerant control strategies for non-linear systems," Lecture Notes in Electrical Engineering, vol. 266, pp. 375-392, 2014.

[18] Y. Wang, Z. Wang, V. Puig, and G. Cembrano, "Zonotopic fault estimation filter design for discrete-time descriptor systems," IFACPapersOnLine, vol. 50, no. 1, pp. 5055-5060, 2017.

[19] Y. Wang, M. Zhou, V. Puig, G. Cembrano, and Z. Wang, "Zonotopic fault detection observer with $H_{-}$performance," in Control Conference (CCC), 2017 36th Chinese. IEEE, 2017, pp. 7230-7235.

[20] H. Hamdi, M. Rodrigues, C. Mechmeche, and N. B. Braiek, "Robust fault detection and estimation for descriptor systems based on multimodels concept," International Journal of Control, Automation and Systems, vol. 10, no. 6, pp. 1260-1266, 2012.

[21] F.-R. López-Estrada, J.-C. Ponsart, C.-M. Astorga-Zaragoza, J.-L. Camas-Anzueto, and D. Theilliol, "Robust sensor fault estimation for descriptor-LPV systems with unmeasurable gain scheduling functions: Application to an anaerobic bioreactor," International Journal of Applied Mathematics and Computer Science, vol. 25, no. 2, pp. 233244, 2015.

[22] F.-R. López-Estrada, J. C. Ponsart, D. Theilliol, and C.-M. AstorgaZaragoza, "Robust $H_{-} / H_{\infty}$ fault detection observer design for descriptor-LPV systems with unmeasurable gain scheduling functions," International Journal of Control, vol. 88, no. 11, pp. 2380 2391, 2015.

[23] B. Marx, D. Koenig, and D. Georges, "Robust fault-tolerant control for descriptor systems," IEEE Transactions on Automatic Control, vol. 49, no. 10, pp. 1869-1876, 2004.

[24] M. Rodrigues, H. Hamdi, N. B. Braiek, and D. Theilliol, "Observerbased fault tolerant control design for a class of LPV descriptor systems," Journal of the Franklin Institute, vol. 351, no. 6, pp. 31043125, 2014.

[25] J. Lunze and T. Steffen, "Control reconfiguration after actuator failures using disturbance decoupling methods," IEEE Transactions on Automatic Control, vol. 51, no. 10, pp. 1590-1601, 2006.

[26] D. Rotondo, F. Nejjari, and V. Puig, "A virtual actuator and sensor approach for fault tolerant control of LPV systems," Journal of Process Control, vol. 24, no. 3, pp. 203-222, 2014.

[27] D. Rotondo, V. Puig, F. Nejjari, and J. Romera, "A fault-hiding approach for the switching quasi-LPV fault-tolerant control of a four-wheeled omnidirectional mobile robot," IEEE Transactions on Industrial Electronics, vol. 62, no. 6, pp. 3932-3944, 2015.

[28] D. Rotondo, F. Nejjari, and V. Puig, "Fault tolerant control of a proton exchange membrane fuel cell using Takagi-Sugeno virtual actuators," Journal of Process Control, vol. 45, pp. 12-29, 2016.

[29] J. H. Richter, W. Heemels, N. van de Wouw, and J. Lunze, "Reconfigurable control of piecewise affine systems with actuator and sensor faults: stability and tracking," Automatica, vol. 47, no. 4, pp. 678-691, 2011.

[30] D. Rotondo, A. Cristofaro, and T. A. Johansen, "Fault tolerant control of uncertain dynamical systems using interval virtual actuators," International Journal of Robust and Nonlinear Control, vol. 28, no. 2, pp. 611-624, 2018.

[31] A. Varga, Solving Fault Diagnosis Problems. Springer, 2017.

[32] M. de Oliveira, J. Bernussou, and J. Geromel, "A new discrete-time robust stability condition," Systems \& Control Letters, vol. 37, no. 4, pp. 261-265, 1999.

[33] J. Löfberg, YALMIP: A Toolbox for Modeling and Optimization in MATLAB, 2004. [Online]. Available: http://users.isy.liu.se/johanl/yalmip

[34] M. Kocvara and M. Stingl, PENBMI User's guide, 2005. [Online]. Available: http://www.penopt.com

[35] MOSEK ApS, The MOSEK optimization toolbox for MATLAB manual. Version 7.1 (Revision 28)., 2015. [Online]. Available: http://docs.mosek.com/7.1/toolbox/index.html 